\title{
BMJ Open Enhancing Prehospital Outcomes for Cardiac Arrest (EPOC) study: sequential mixed-methods study protocol in Michigan, USA
}

\author{
Rama A Salhi (D) , ${ }^{1,2}$ Sydney Fouche, ${ }^{2}$ Peter Mendel, ${ }^{3}$ Christopher Nelson, ${ }^{3}$ \\ Michael D Fetters, ${ }^{4}$ Timothy Guetterman, ${ }^{4}$ Jane Forman, ${ }^{2,5}$ Wilson Nham, ${ }^{2}$ \\ Jason E Goldstick, ${ }^{2}$ Jessica Lehrich, ${ }^{2}$ Bill Forbush, ${ }^{6}$ Samantha lovan, ${ }^{2}$ Antony Hsu, ${ }^{7}$ \\ Theresa A Shields, ${ }^{8}$ Robert Domeier, ${ }^{7}$ Claude M Setodji, ${ }^{3}$ Robert W Neumar, ${ }^{8}$ \\ CARES Surveillance Group, Brahmajee K Nallamothu, ${ }^{9}$ Mahshid Abir ${ }^{2,8}$
}

To cite: Salhi RA, Fouche $S$, Mendel P, et al. Enhancing Prehospital Outcomes for Cardiac Arrest (EPOC) study: sequential mixedmethods study protocol in Michigan, USA. BMJ Open 2020;10:e041277. doi:10.1136/ bmjopen-2020-041277

Received 03 June 2020 Revised 30 October 2020 Accepted 02 November 2020

Check for updates

(c) Author(s) (or their employer(s)) 2020. Re-use permitted under CC BY-NC. No commercial re-use. See rights and permissions. Published by BMJ.

For numbered affiliations see end of article.

Correspondence to Dr Mahshid Abir; mahshida@med.umich.edu

\section{ABSTRACT}

Introduction Out-of-hospital cardiac arrest (OHCA) is a common, life-threatening event encountered routinely by first responders, including police, fire and emergency medical services (EMS). Current literature suggests that there is significant regional variation in outcomes, some of which may be related to modifiable factors. Yet, there is a persistent knowledge gap regarding strategies to guide quality improvement efforts in OHCA care and, by extension, survival. The Enhancing Prehospital Outcomes for Cardiac Arrest (EPOC) study aims to fill these gaps and to improve outcomes.

Methods and analysis This mixed-methods study includes three aims. In aim I, we will define variation in OHCA survival to the emergency department (ED) among EMS agencies that participate in the Michigan Cardiac Arrest Registry to Enhance Survival (CARES) in order to sample EMS agencies with high-survival and low-survival outcomes. In aim II, we will conduct site visits to emergency medical systems-including 911/dispatch, police, non-transport fire, and EMS agencies-in approximately eight high-survival and low-survival communities identified in aim I. At each site, key informant interviews and a multidisciplinary focus group will identify themes associated with high OHCA survival. Transcripts will be coded using a structured codebook and analysed through thematic analysis. Results from aims I and II will inform the development of a survey instrument in aim III that will be administered to all EMS agencies in Michigan. This survey will test the generalisability of factors associated with increased OHCA survival in the qualitative work to ultimately build an EPOC Toolkit which will be distributed to a broad range of stakeholders as a practical 'how-to' guide to improve outcomes.

Ethics and dissemination The EPOC study was deemed exempt by the University of Michigan Institutional Review Board. Findings will be compiled in an 'EPOC Toolkit' and disseminated in the USA through partnerships including, but not limited

\section{Strengths and limitations of this study}

- The Enhancing Prehospital Outcomes for Cardiac Arrest (EPOC) study is a multiphase mixed-methods approach combining quantitative and qualitative data to identify challenges and effective strategies for prehospital out-of-hospital cardiac arrest (OHCA) care along the care continuum.

- The mixed-methods study design provides a comprehensive approach to understanding complex systems of care and elucidating best practices for OHCA survival.

- At completion of the study, we will be able to provide an 'EPOC Toolkit' that would provide strategies for $\mathrm{OHCA}$ best practices with the goal of improved survival outcomes.

- Use of administrative data to complete a secondary data analysis; study limitations include quality of coding, missingness and residual confounding.

- The qualitative analysis component of our study is exploratory and may be limited by the single time point of data collection, as well as possible social desirability response bias.

to, policymakers, EMS leadership and health departments.

\section{INTRODUCTION}

Out-of-hospital cardiac arrest (OHCA) is a common, life-threatening event that is associated with poor patient outcomes. Among those experiencing OHCA, approximately $30 \%$ of patients survive to hospital admission, and roughly $10 \%$ are ultimately discharged alive. ${ }^{2}$ In addition to the low survival rates, it is estimated that the total aggregate cost of OHCA care in the USA is $\$ 33$ billion annually. ${ }^{3}$ Despite generally poor outcomes, some communities have been consistently more 
successful in responding to and treating OHCA than others. ${ }^{4-6}$ By some estimates, a remarkable fivefold difference in survival exists across US communities. ${ }^{4}$

Emergency medical services (EMS) have been recognised as a critical component for improving OHCA survival; current recommendations for improving prehospital OHCA care are focused on specific care processes, such as high-quality resuscitation and postarrest care. ${ }^{6}$ The 2015 Institute of Medicine (IOM) report ${ }^{6}$ recognises the 'chain of survival' - which includes five interdependent links-as an operational framework for assessing EMS response to OHCA: (1) early recognition of cardiac arrest and activation of emergency response, (2) early cardiopulmonary resuscitation (CPR), (3) rapid defibrillation, (4) effective advanced cardiac life support and (5) integrated postresuscitative care. ${ }^{7}$ Given that first responders (including EMS, fire and police) are the first on the scene of a cardiac arrest, the importance of initiating CPR, applying an automated external defibrillator (AED), and effective communication among first-responder entities and 911 dispatchers is clear. ${ }^{8-12}$ Proposed care delivery models and processes that are associated with improved OHCA care include 911 dispatcherassisted bystander $\mathrm{CPR},{ }^{12}{ }^{13}$ use of computer-assisted dispatching, ${ }^{14}{ }^{15}$ improved coordination among ambulance crews, ${ }^{6}{ }^{16}$ delivering high-performance CPR, ${ }^{7}{ }^{17}$ use of technology shown to improve prehospital care for OHCA, ${ }^{18}$ improved provision of elements of postarrest care before or during transport, ${ }^{19}$ improved transitions of care between first responders and hospitals, ${ }^{61120}$ continuous quality improvement efforts, ${ }^{21}{ }^{22}$ and promoting a culture of excellence through exemplary leadership and accountability. ${ }^{23}$

Although these measures are associated with improved outcomes in isolated reports, it is still unclear how best to design strategies that can be uniformly implemented in emergency medical systems across different communities. Additionally, each community may deal with different challenges in the implementation of these strategies due to its specific needs and resources. For example, existing data indicate that EMS systems' effectiveness may vary based on resource availability and transport times (eg, long distance in rural areas and traffic in urban areas may delay response), ${ }^{6}{ }^{24}$ EMS staffing models, ${ }^{25-27}$ interstakeholder coordination (or fragmentation) and EMS oversight. $^{6} 21222526$ Furthermore, some communities may be reluctant to call 911 (e.g., undocumented immigrants and those in high-crime neighbourhoods), which can delay response and raise access issues for vulnerable populations. ${ }^{28-31}$

In 2013, the IOM and the National Academy of Engineering published the discussion paper 'Bringing a Systems Approach to Health', which recommends considering the relationships between the various parts of healthcare delivery to better understand the elements that influence health outcomes and produce better health at lower costs. ${ }^{16}$ An integrative systems approach has been recognised as critical to cardiac resuscitation. ${ }^{32}$ Similarly, the importance of an iterative approach is highlighted by the integration of continuous quality improvement programmes in EMS agencies in the USA. Despite this, there remains a paucity of systems-based rigorous mixedmethodology approaches as a lens through which to evaluate the complexities of prehospital cardiac arrest care. This is a relatively novel approach to investigating the interaction of EMS agencies with the other responding entities that provide care before and after EMS response, including community bystanders, dispatch, other first responders and hospitals. Accordingly, the Enhancing Prehospital Outcomes for Cardiac Arrest (EPOC) study will lay the foundation for future quality improvement efforts in OHCA by identifying, understanding and validating best practices adopted by high-survival emergency medical systems. Similarly, describing such strategies across the continuum of care will elucidate potential barriers to implementation of best practices. In this paper, we describe the content and conceptual framework of the multiphase, mixed-methods study we employed to accomplish this goal.

\section{Aims and objectives}

In this project, we use a multiphase mixed-methods approach (figure 1) combining quantitative data analyses with qualitative data from key informant interviews and focus groups to identify challenges and effective strategies for prehospital OHCA care along the care continuum. We chose a mixed-methods approach because prehospital care is multifaceted and complex. Findings could be affected by many stakeholders-such as state and local governments, hospitals, 911/dispatch, first responders (including police and fire), EMS and others-and OHCA outcomes often depend on multiple care processes, as well as organisational cultures around care delivery that shape group interactions. These aspects of prehospital care are difficult to measure in purely quantitative studies and can benefit from qualitative approaches that are suited to understanding complex systems and elucidating what happens, how it happens and why. ${ }^{33-35}$

Accordingly, the aims of this study were

- To identify EMS agencies with high-OHCA and lowOHCA survival rates (aim I).

- To define best practices and barriers within emergency medical systems associated with OHCA survival (aim II).

- To validate factors associated with high survival through developing and administering a novel survey to EMS agencies statewide ( aim III).

\section{Patient and public involvement}

Cardiac arrest survivors and their family members were involved at the inception of the study and provided insights to the study team regarding their experiences. In addition, study team members were also involved in an ongoing OHCA learning community involving community members, survivors, first responders and other healthcare providers. The public, through interviews and 


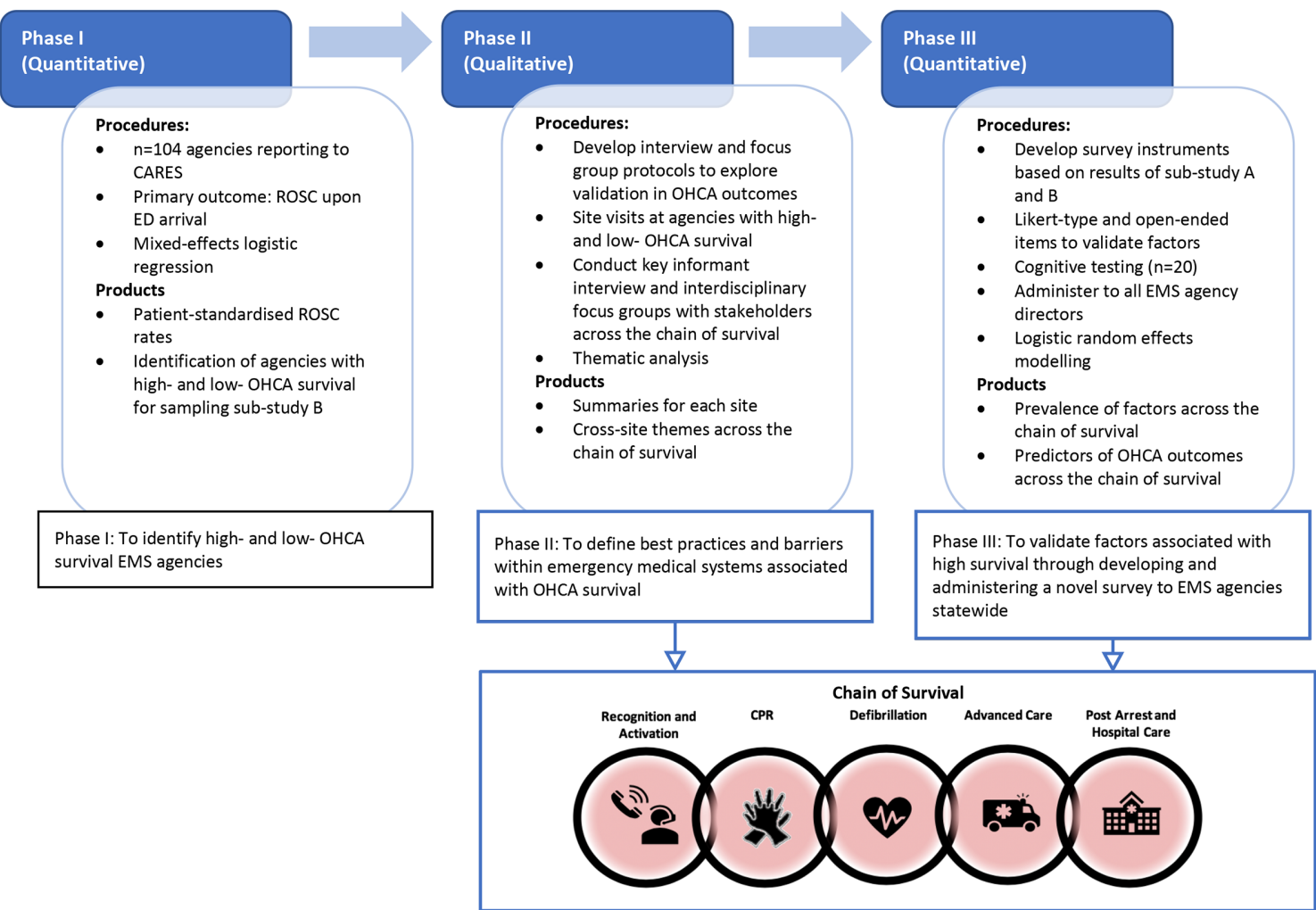

Figure 1 Multiphase mixed-methods approach to identifying high OHCA survival best practices. CARES, Cardiac Arrest Registry to Enhance Survival; CPR, cardiopulmonary resuscitation; ED, emergency department; EMS, emergency medical services; OHCA, out-of-hospital cardiac arrest; ROSC; return of spontaneous circulation.

focus groups involving police departments, fire departments and EMS, were central throughout aims II and III.

\section{METHODS/DESIGN}

This study was designed to examine OHCA across the continuum of care by examining the structures, processes and interdependencies in prehospital care delivery using a multiphase mixed-methods approach. Specifically, we initiate our study in aim I with a quantitative component to elucidate trends in overall survival between EMS agencies. Using these identified EMS agencies then allows us to intentionally sample those EMS agencies for in-depth qualitative assessments in aim II. As such, in aim I, we will elucidate EMS agencies with high-OHCA and low-OHCA survival rates and identify a sample for more focused study. In aim II, we will elucidate best practices and barriers to implementation by examining EMS agencies with high-OHCA and low-OHCA survival rates using a qualitative case study approach to link specific site characteristics and performance, with the perspectives of frontline operations through key informant interviews and focus groups. Based on the factors identified as important by multiple key stakeholders, we will gain new insights about achieving a successful prehospital OHCA response. In aim III, we propose to validate and refine the quantitatively and qualitatively derived model for maximising survival by distributing a state-wide survey to assess the importance of the identified factors and their predictivity for improved survival.

\section{Conceptual model}

The study's overall conceptual framework is displayed in figure 2. As illustrated in the model, advancing OHCA outcomes must account for patient and community characteristics, EMS agency organisational characteristics and interorganisational characteristics. Additionally, the model informs the primary outcome of interest for the EPOC study, namely, the return of spontaneous circulation (ROSC) with pulse on ED arrival. This outcome measure best encompasses the totality of prehospital care provided to patients who have OHCA. The model further delineates secondary outcomes, namely, any ROSC in the prehospital setting, survival to hospital admission, survival to hospital discharge and survival with good neurological function.

\section{Setting}

The state of Michigan will serve as the setting for this research, with study dates spanning August 2017 to a projected end date of July 2021. The state of Michigan provides an ideal location to conduct this research as there are significant variations in the OHCA survival rates, which suggests the practices of agencies with high OHCA survival could inform improvements among those with lower survival. ${ }^{36}$ As a Midwest US state, Michigan has urban, suburban and rural locations that allow for 


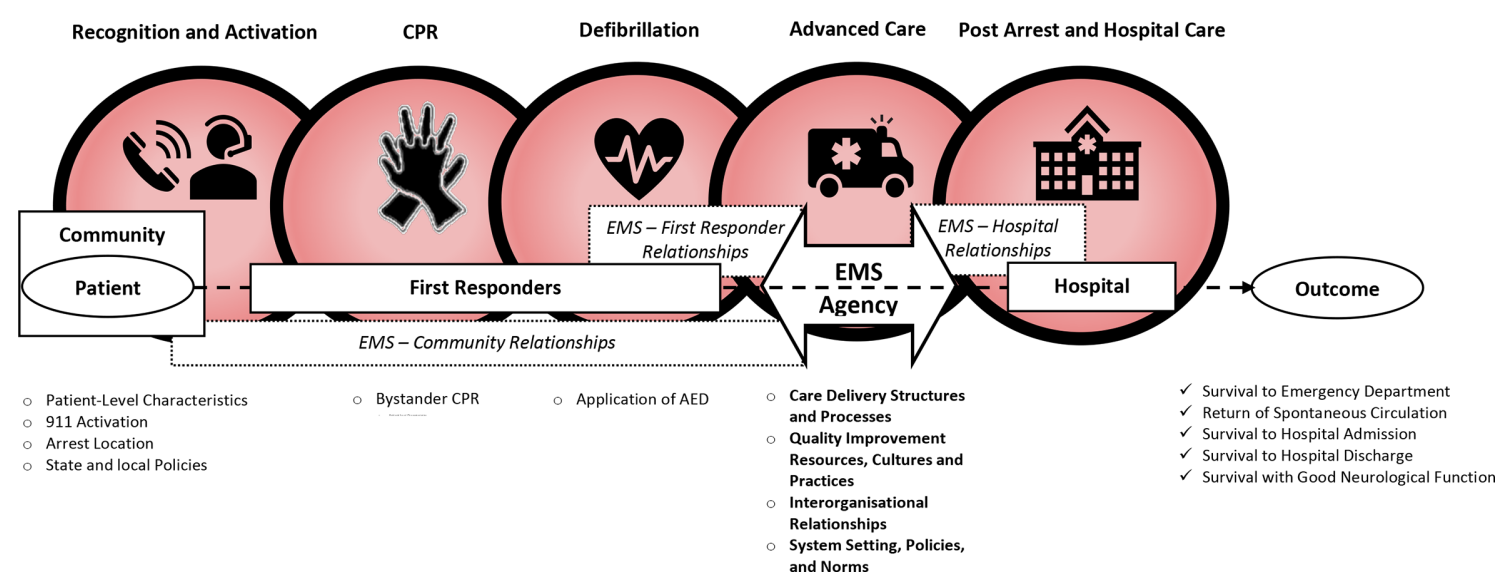

Figure 2 Conceptual model of factors influencing out-of-hospital cardiac arrest outcomes across the chain of survival. AED, automated external defibrillator; CPR, cardiopulmonary resuscitation; EMS, emergency medical services.

examination of population density on OHCA outcomes. Further, there are a variety of EMS structures that reflect the service models seen in the USA, allowing for exploration of a range of implementation strategies. Finally, Michigan operates a large OHCA registry-Michigan Cardiac Arrest Registry to Enhance Survival (MI-CARES).

Michigan is one of 26 state registries contributing to CARES nationally. Through 2017, there were 136 EMS agencies and 110 hospitals contributing data to MI-CARES, which equates to coverage of approximately $80 \%$ of the state's population. ${ }^{37}$ Michigan also has a fulltime state coordinator who is responsible for record auditing, overseeing data collection, and training EMS agencies and hospitals on CARES data entry. In the context of prehospital resuscitation care, MI-CARES contains a broad-based sample of EMS agencies in urban, suburban and rural areas in MI with representation from various regions of the state. ${ }^{38}$ Using this information in conjunction with primarily collected qualitative data, we seek to elucidate each of the previously described aims as outlined further.

Aim I: identification (ID) of EMS agencies with high-OHCA and low-OHCA survival rates

Data source

The CARES dataset is the largest OHCA registry in the USA. The programme has expanded nationally to include 26 statewide registries with more than 50 community sites in 16 additional states. CARES represents a catchment area of approximately 135 million people or $40 \%$ of the US population. The secure web database has software that collects and links data sources from EMS providers, dispatch centres and hospitals to create a single deidentified record for each OHCA event. CARES was developed to help communities determine standard outcome measures for OHCA to allow for quality improvement and benchmarking, as well as compare their EMS system performance to deidentified aggregate statistics at the local, state or national level. ${ }^{3739}$

Given the significant role of community-level variables in cardiac arrest outcomes, individual-level data will be augmented with community-level data. For the years under study, we will obtain data from the American Community Survey to adjust for median age, racial demographic makeup (proportion white, black, Asian, mixed race and other race), proportion of male residents, average household size, unemployment, per cent population with general equivalency diploma (GED) or higher, per cent population greater than 25 years old, median household income, population density per square mile and land area. For each county, we will also calculate aggregate proportions of bystander CPR and AED use. A calendar year covariate will also be included to adjust for temporal trends.

\section{Study cohort/variables}

Our first goal was to identify EMS agencies with high and low survival rates, to provide descriptive information and to inform sampling strategies in the subsequent substudies. We will use previously collected data on all EMS-treated OHCA from agencies with five or more of such incidents annually in MI-CARES for years 2014-2017. A total of 104 agencies covering more than 7.4 million lives and 21100 patients who had OHCA met the inclusion criteria. EMS agencies with fewer than 5 OHCA incidents over the 3-year study period will be excluded from the analysis. This cut-off will still allow for the inclusion of agencies in rural and suburban areas-with few OHCA incidentsand will also permit evaluation of high and low census agencies in aim II, which may face unique challenges in treating OHCA. Using these data, we will identify EMS agencies with high and low survival rates participating in MI-CARES.

\section{Outcome variables}

The primary outcome of interest in the EPOC study is ROSC with pulse on ED arrival, analysed as a binary outcome. This was chosen as the primary outcome as we believe it represents the final results of the complex strategies required to achieve high performance in OHCA care by the prehospital system. Additional cardiac arrest outcomes considered in this study included any ROSC 
in the prehospital setting, survival to hospital admission, survival to hospital discharge, in-hospital mortality and survival with good neurological outcomes. While all represent important clinical and patient-centred outcomes, they additionally are limited when considering them as a primary prehospital outcome. Any ROSC in the prehospital setting provides us with a prehospital-specific outcome but fails to capture the entire prehospital timeline. Alternatively, survival to hospital admission, hospital discharge and good neurological outcomes are heavily influenced by ED and hospital-based interventions, potentially limiting our ability to narrow the focus specifically to the prehospital providers' role in OHCA. As such, ROSC with pulse on ED arrival was chosen as the primary outcome.

Given the continued importance of the consideration of the additional outcomes, they will be analysed as secondary outcome measures. Secondary outcomes will include binary indicators of (1) any ROSC in the prehospital setting; (2) survival to hospital admission (defined as survival to admission to the intensive care unit, cardiac care unit or the medical floor); (3) survival to hospital discharge (defined as survival to discharge to home, a rehabilitation facility, a long-term care facility or a nursing home); (4) survival with good neurological outcome (defined as a cerebral performance category score of 1 or 2 at the time of discharge); and (5) in-hospital mortality. These secondary outcomes represent more immediate outcomes (e.g., ROSC), as well as longer-term outcomes (e.g., in-hospital mortality).

\section{Analysis}

Using data from MI-CARES, we will evaluate variation in OHCA survival among MI-CARES-participating EMS agencies. A priori power calculations were completed using MI-CARES data from 2015. Based on these data, we anticipated approximately 15000 OHCA cases across 63 agencies. Given the large number of cases, the primary limitation here for estimating the model parameters is the number of clusters. Prior work indicates that having more than 50 clusters, even with only 100 or fewer individuals per cluster, will result in accurate fixed-effects estimates and variance components. We augmented this with a simulation study using 63 clusters and estimated the random-effects variance. This demonstrated excellent ability to recover the estimated random-effects variance, confirming that our sample size is sufficient to characterise between-agency variation.

For our primary analysis, we will calculate patientstandardised ROSC rates for OHCA across EMS agencies from 2014 to 2017, adjusting for variables, including age, gender, race, location of arrest, witnessed arrest, shockable rhythm, bystander CPR, AED use, and community-level demographics. Included in our model will be previously identified community-level variables as described previously. Using a mixed-effects logistic regression model, we will then identify EMS agencies with a greater than $90 \%$ predicted probability of having been in the upper and lower quartiles in Michigan for the primary outcome of ROSC with pulse on arrival to ED. Among the identified agencies, we will invite up to four EMS agencies with high survival and four EMS agencies low survival to participate in aim II site visits.

Aim II: defining best practices and barriers within emergency medical systems associated with OHCA survival

Study population sampling

To develop a deeper understanding of the systems-level and organisational factors contributing to the expected differences in OHCA outcomes between EMS agencies from aim I, we will qualitatively explore variation in characteristics, practices and strategies across the emergency medical systems in the sampled communities. To accomplish this, we will conduct site visits to conduct key informant interviews and interdisciplinary focus groups at the sampled MI-CARES-participating EMS agencies and their associated emergency medical system stakeholder groups (i.e., 911/dispatch, fire and police).

Agencies identified as being in the upper or lower end of the residual OHCA survival distribution will be invited to participate in site visits by the MI-CARES state coordinator. The performance category of agencies will not be disclosed to key informant interview and focus group participants. In addition to OHCA survival, EMS agencies will be sampled based on geography (based on the eight state trauma regions), rurality and population density.

\section{Data collection}

Prior to each site visit, we will conduct a baseline survey of the EMS agencies. The content of these brief electronic surveys, administered by email, will include the prevalence and categorisation of strategies used by agencies for treating OHCA. The survey will also examine an agency's existing infrastructure to review OHCA cases, certification of EMS staff for basic and advanced life support, system of dispatching and monitoring patients who have OHCA and cardiac arrest processes of care. This survey has been included in online Appendix A.

During each two-day site visit, we will conduct key informant interviews with field staff, mid-level managers and leadership from each stakeholder group (ie, 911/ dispatch, police, non-transport fire and EMS agencies), as well as a multidisciplinary focus group to identify facilitators and barriers to achieving high OHCA survival in the prehospital setting. The key informant interviews will explore in depth stakeholder-specific factors relevant to OHCA care delivery. Information from these interviews will be used to guide multistakeholder focus group discussions at the end of day two. Each focus group will include 10-12 participants representing various parts of the emergency medical system, including EMTs, paramedics, fire and police personnel, dispatchers/call takers and ED nurses. These multidisciplinary focus groups will be conducted at each EMS agency to promote interactions that facilitate understanding of the care system, which involves overlap of EMS operations and care delivery 
across prehospital care organisations. We will conduct follow-up key informant interviews for each site with individuals identified as OHCA or EMS system 'champions'.

The same interview guide will be used for key informant interviews and focus groups. The interview guide will begin with general, open-ended questions followed by questions focused on OCHA-related strategies and processes. Issues related to transitions of care/hand-offs among responding entities will be explored. Specific probes concerning initiatives targeted at treating OHCA will follow. For each initiative, respondents will be asked to describe specific challenges, surprises, and successes in implementing change in their organisation's practices and procedures regarding OHCA. In addition, respondents will be asked about their experiences with quality improvement efforts (e.g., organisational goals and use of data feedback). Finally, respondents will be asked questions about communication and organisational culture to understand the working environment and interorganisational relationships (see online Appendix B for interview guide). Interviews will be audio-recorded and transcribed by independent professional transcriptionists to enhance accuracy.

\section{Analysis}

Transcripts will be coded using a structured codebook and will be analysed through thematic analysis. Analysis of preliminary data from 4 focus groups and 10 key informant interviews with medical control authorities (MCAs) and stakeholders in Michigan showed that themes were not saturated. As such, we will aim to conduct up to 10 site visits until we achieve saturation of themes. We will conduct within-site (i.e., emergency medical system) then cross-site analyses to identify themes that will inform survey development (aim III) and describe best practices, barriers and facilitators to implementing those practices.
For each site, researchers from the study team will review the key informant interviews and focus group transcripts to identify themes. We will use our preliminary taxonomy of factors (table 1) as a priori codes and conduct an inductive analysis to refine our coding scheme and to identify emergent codes.

Initially, a sample of transcripts will be coded by three coders. The coders will meet periodically to share coded transcripts, and the codebook will be developed through an iterative process. Remaining transcripts will be coded by one coder. MAXQDA, ${ }^{40}$ a qualitative analysis software, will be used to facilitate data coding. After all data have been coded, we will run reports for each code in MAXQDA, which will be reviewed by the team to discuss potential themes and interpretations. We will then create summaries for each site by code, and, to facilitate crosssite analysis, will use ordered matrices to account for all relevant data, identify patterns (eg, relationships between factors and outcomes) and develop cross-site themes. ${ }^{41}$

\section{Aim III: validation of factors associated with high survival through developing and administering a novel survey to EMS agencies statewide \\ Instrument development}

The survey development will be informed by the study conceptual model (figure 2) and prior phases of the study with inclusion of emerging themes from the qualitative analysis. We will use a mixed-methods approach to survey instrument development, which will include pilot testing and cognitive testing of the instrument. This survey will consist of a combination of Likert scale and open-ended questions to assess the prevalence and importance of previously identified factors. Whenever possible, we will develop elements of this survey from any related, previously validated surveys from the fields of resuscitation science, EMS and organisational science. A number of

Table 1 Preliminary taxonomy for codebook factors and dimensions

\begin{tabular}{ll}
\hline Factor & Dimensions \\
\hline Organisational characteristics & Community, first responder, EMS, hospital structure or culture. \\
Leadership & Commitment of clinical or public safety leadership. \\
& Profsional credibility of leaders. \\
& Secific behaviours of leaders in QI or care delivery. \\
Administrative support & Provision of necessary financial and physical resources. \\
& Engagement of leadership in QI and staff relationships. \\
Care delivery strategies & Fostering QI culture. \\
QI practices & Types of interventions across OHCA spectrum. \\
& Timeliness and perceived accuracy of data. \\
& Use of benchmarks in data feedback. \\
& Resources for implementing and sustaining QI programmes. \\
Stakeholder relationships & Relationships between hospitals and EMS, EMS and first responders, first \\
& responders and the community.
\end{tabular}

EMS, emergency medical services; OHCA, out-of-hospital cardiac arrest; QI, quality improvement. 
questions will be directed at understanding the characteristics of each agency (e.g., ownership, number of basic life support and advanced life support units, number of field staff and profit status) to include as covariates in analytical models.

We will conduct cognitive interviews and field testing of the developed survey with a small sample of agencies that did not participate in its development to receive feedback on content and design. Using that information, the study team will refine the final survey instrument through an iterative process in collaboration with content and methodological experts. Through cognitive testingone-on-one interviews with EMS agency directors-the draft survey will be finalised. We will conduct a total of 20 cognitive interviews using the final survey via telephone call with EMS agency representatives across Michigan, including administrators, EMTs and paramedics, and quality improvement coordinators among other roles. Participants will receive $\$ 20$ as compensation for their time.

The final survey will be developed in Qualtrics XM and will be distributed to all transporting EMS agency directors via email. We anticipate that survey completion will take $30 \mathrm{~min}$. Respondents will be compensated $\$ 20$ for their time. To increase the survey response rate, we will leverage previous relationships with the Michigan Department of Health and Human Services (MDHHS) to encourage survey response by EMS agencies through the states MCAs-entities tasked with EMS oversight. Surveys completed by participating EMS agencies will be tracked with uniquely identified provider-level CARES registry site IDs. Electronic reminders will be sent to EMS administrators with financial incentives for participation.

\section{Analysis}

Our primary analytical tool will be a logistic random-effects model similar to the one proposed in aim I, augmented with the addition of the agency-level variables developed. Diagnostic analyses will be performed to identify problems, including collinearity, contaminated inference, non-linear main effects and interactions between predictors. We will use the results to examine the overall prevalence of key factors (identified in aim II) in EMS agencies across the state and determine to what extent the newly developed measure explained between-agency variation observed in aim I. Then for MI-CARES-participating EMS agencies, survey responses will be linked to the agency's MI-CARES data to assess the association of each factor with improved OHCA outcomes in cross-sectional analyses. This will serve to validate strategies identified in aim II most strongly associated with improved outcomes among a broader group of EMS agencies in Michigan. Identified best practices will be added into the EPOC Toolkit for dissemination.

Power calculations were conducted using the rate of non-traumatic OHCA survival of $10.5 \%$ based on CARES data. ${ }^{1}$ Using 2015 CARES data, we prospectively estimated a nominal sample size of 210 cases per agency over the anticipated study period. We conducted a power calculation that conservatively assumes an effective sample size of 40 (less than 20\%) of the nominal sample size, making this calculation conservative. Using mixed-effects logistic regression clustering at the county level, we estimated (after adjusting for covariates) a within-county random-effects variance of only $a=0.06$. Simulation-based estimates of minimum detectable ORs ( $80 \%$ power) for OHCA incidence and survival for a dichotomous quality performance metric endorsed by $10 \%, 25 \%$ and $50 \%$ of agencies were $1.23,1.16$ and 1.13 , respectively. These estimates are within the limit of clinically relevant findings for OHCA care and, even given the conservativeness of this calculation, the detectable effect sizes are still relatively small.

\section{Ethics and dissemination}

We obtained approval from the CARES national office to use data from Michigan. Study aims I, II and III were reviewed by the University of Michigan Institutional Review Board and were deemed exempt (HUM00142906 and HUM00168850). A verbal informed consent to participate in the study was obtained from all participants and is available in the interview guide in online Appendix B and was provided to all participants.

To disseminate our findings, we will develop the 'EPOC Toolkit' that includes strategies associated with high survival to the ED following OHCA. We will then use multiple approaches to share these findings in order to promote best practices for OHCA in communities across the USA. This toolkit will include possible facilitators and barriers to implementing such efforts and the evidence base for each.

Our primary approach will be to work with state and national partners to develop a national quality programme. Building on partnerships developed throughout the first several years of the project, we will work with state policymakers, EMS leadership and the MDHHS to disseminate the EPOC Toolkit through a conference for first responder practitioners in year four.

Beyond Michigan, we will work with the national CARES programme, as well as the American Heart Association and other stakeholders invested in improving cardiac arrest outcomes. Through EPOC and future studies, our dissemination plan will (1) highlight the evidence base and practical, actionable strategies for improving OHCA survival; (2) align OHCA care with quality improvement goals; (3) recruit local opinion leaders in prehospital care; (4) leverage key sponsors to construct provider networks to share learning opportunities; and (5) evaluate the end results of these efforts using CARES.

\section{DISCUSSION}

OHCA remains a common, life-threatening condition with persistent outcome gaps across communities. While many previous studies have adopted a piecemeal approach, focusing on evaluating one or two interventions 
at a time, providing high-quality prehospital care for OHCA is multifaceted and complex, affecting several stakeholders and disciplines. It depends on multiple care processes, as well as organisational cultures that guide interactions across groups. These aspects of care are difficult to measure in quantitative studies but can be assessed and understood with qualitative methods which we incorporate as a core component of our study. The promise of the use of mixed-methods for advancing care can be illustrated by examples from previous research. For example, a sequential mixed-methods approach has previously been used successfully to identify key strategies to improve in-hospital cardiac arrest response, to reduce door-to-balloon times for primary percutaneous coronary intervention $^{42-46}$ and to improve outcomes after acute myocardial infarction. ${ }^{47} 48$

Here we directly examine strategies adopted by EMS agencies and emergency medical systems to target care processes, and build on prior research that has previously relied on enumerating global processes of care (e.g., time to defibrillation) or structural variables (e.g., geographical location and urbanicity) and their association with OHCA outcomes. As these associations do not always provide actionable insights into how OHCA care can be improved in the prehospital setting, ${ }^{49}$ we attempt to identify here a broader range of modifiable elements to improve patient outcomes. Through our proposed approach, we will account for the multidisciplinary nature and community context of OHCA care in the prehospital setting and incorporate the role of all key stakeholders: the community, 911/dispatch, first responders (police and fire), EMS agencies and hospitals. ${ }^{50}$

\section{Author affiliations}

${ }^{1}$ Department of Emergency Medicine, University of Michigan, Ann Arbor, Michigan, USA

${ }^{2}$ Acute Care Research Unit, Institute for Healthcare Policy and Innovation, University of Michigan, Ann Arbor, Michigan, USA

${ }^{3}$ RAND Corporation, Santa Monica, California, USA

${ }^{4}$ Department of Family Medicine, University of Michigan, Ann Arbor, Michigan, USA

${ }^{5}$ Center for Clinical Management Research, Veterans Affairs Health System, Ann

Arbor, Michigan, USA

${ }^{6}$ City of Alpena Fire Department, Alpena County EMS, City of Alpena, Alpena, Michigan, USA

${ }^{7}$ Department of Emergency Medicine, Saint Joseph Mercy Health System, Ann Arbor, Michigan, USA

${ }^{8}$ Department of Emergency Medicine, University of Michigan Health System, Ann Arbor, Michigan, USA

${ }^{9}$ Division of Cardiovascular Diseases and the Department of Internal Medicine, University of Michigan Health System, Ann Arbor, Michigan, USA

Contributors RAS participated in data collection and analysis, as well as drafting and editing of the manuscript. SF, SI and WN participated in study coordination, data collection and analysis, as well as editing of the manuscript. JF, TG, JEG and JL participated in design of the study, data collection and analysis, as well as editing of the manuscript. PM, CN, CMS, JG, AH, BF, MDF, TAS, RD and RWN contributed to conception and design of the study, as well as drafting and editing of the manuscript. MA and BN contributed to conception and design of the study, obtaining funding, study execution oversight, as well as drafting and editing of the manuscript. All authors have read and approved the final manuscript.
Funding This work was supported by the National Heart, Lung, and Blood Institute, and all listed authors received funding from this grant (grant number: 5R01HL137964). The funding sources had no role in the design of the protocol.

Competing interests During the study period, MA received funding from the American Heart Association for the Michigan-Resuscitation Innovation and Science Enterprise, a collaboration focused on improvement of neurological outcomes after cardiac arrest.

Patient consent for publication Not required.

Provenance and peer review Not commissioned; externally peer reviewed.

Supplemental material This content has been supplied by the author(s). It has not been vetted by BMJ Publishing Group Limited (BMJ) and may not have been peer-reviewed. Any opinions or recommendations discussed are solely those of the author(s) and are not endorsed by BMJ. BMJ disclaims all liability and responsibility arising from any reliance placed on the content. Where the content includes any translated material, BMJ does not warrant the accuracy and reliability of the translations (including but not limited to local regulations, clinical guidelines, terminology, drug names and drug dosages), and is not responsible for any error and/or omissions arising from translation and adaptation or otherwise.

Open access This is an open access article distributed in accordance with the Creative Commons Attribution Non Commercial (CC BY-NC 4.0) license, which permits others to distribute, remix, adapt, build upon this work non-commercially, and license their derivative works on different terms, provided the original work is properly cited, appropriate credit is given, any changes made indicated, and the use is non-commercial. See: http://creativecommons.org/licenses/by-nc/4.0/.

ORCID iD

Rama A Salhi http://orcid.org/0000-0002-6533-5878

\section{REFERENCES}

1 Chan PS, McNally B, Tang F, et al. Recent trends in survival from out-of-hospital cardiac arrest in the United States. Circulation 2014;130:1876-82.

2 Coute RA, Nathanson BH, Panchal AR, et al. Disability-Adjusted life years following adult out-of-hospital cardiac arrest in the United States. Circ Cardiovasc Qual Outcomes 2019;12:e004677.

3 Kida K, Ichinose F. Preventing ischemic brain injury after sudden cardiac arrest using no inhalation. Crit Care 2014;18:212-6.

4 Nichol G, Thomas E, Callaway CW, et al. Regional variation in out-of-hospital cardiac arrest incidence and outcome. JAMA 2008;300:1423-31.

5 Zive DM, Schmicker R, Daya M, et al. Survival and variability over time from out of hospital cardiac arrest across large geographically diverse communities participating in the resuscitation outcomes Consortium. Resuscitation 2018;131:74-82.

6 Graham R, McCoy MA, Schultz AM. Strategies to improve cardiac arrest survival: a time to act. Institute of medicine. Washington, DC: National Academies Press, 2015. http://www.nationalacademies.org/ hmd/Reports/2015/Strategies-to-Improve-Cardiac-Arrest-Survival. aspx

7 Berg RA, Hemphill R, Abella BS, et al. Part 5: adult basic life support. Circulation 2010;122:S685-705.

8 White RD, Bunch TJ, Hankins DG. Evolution of a community-wide early defibrillation programme experience over 13 years using police/fire personnel and paramedics as responders. Resuscitation 2005;65:279-83.

9 Mosesso VN, Davis EA, Auble TE, et al. Use of automated external defibrillators by police officers for treatment of out-of-hospital cardiac arrest. Ann Emerg Med 1998;32:200-7.

10 Kellermann AL, Hackman BB, Somes G, et al. Impact of firstresponder defibrillation in an urban emergency medical services system. JAMA 1993;270:1708-13.

11 Husain S, Eisenberg M. Police AED programs: a systematic review and meta-analysis. Resuscitation 2013;84:1184-91.

12 Roppolo LP, Westfall A, Pepe PE, et al. Dispatcher assessments for agonal breathing improve detection of cardiac arrest. Resuscitation 2009;80:769-72.

13 Caffrey SL, Willoughby PJ, Pepe PE, et al. Public use of automated external defibrillators. N Engl J Med 2002;347:1242-7.

14 Swor RA, Hoelzer M. A computer-assisted quality assurance audit in a multiprovider EMS system. Ann Emerg Med 1990;19:286-90.

15 Stewart RD, Burgman J, Cannon GM, et al. A computer-assisted quality assurance system for an emergency medical service. Ann Emerg Med 1985;14:25-9. 
16 Kaplan G, Bo-Linn G, Carayon P, et al. Bringing a systems approach to health. NAM Perspectives 2013;3.

17 Beesems SG, Berdowski J, Hulleman M, et al. Minimizing preand post-shock pauses during the use of an automatic external defibrillator by two different voice prompt protocols. A randomized controlled trial of a bundle of measures. Resuscitation 2016;106:1-6.

18 Ko PC-I, Chen W-J, Lin C-H, et al. Evaluating the quality of prehospital cardiopulmonary resuscitation by reviewing automated external defibrillator records and survival for out-of-hospital witnessed arrests. Resuscitation 2005;64:163-9.

19 Pinchalk ME. Managing post cardiac arrest syndrome in the prehospital setting. JEMS 2010.

20 Landman AB, Spatz ES, Cherlin EJ, et al. Hospital collaboration with emergency medical services in the care of patients with acute myocardial infarction: perspectives from key hospital staff. Ann Emerg Med 2013;61:185-95.

21 NHTSA. National highway traffic safety administration (NHTSA): advancing EMS systems 2016, 2016. Available: http://www.ems.gov/ advancing-ems-systems.html [Accessed 22 Aug 2019].

22 NHTSANO. Emergency medical services performance measures: recommended attributes and indicators for system and service performance. Washington, DC: National Highway Traffic Safety Administration (NHTSA), 2009.

23 Institute for Healthcare Improvement. Optimizing patient flow: moving patients smoothly through acute care settings. Available: http://www.ihi.org:80/resources/Pages/lHIWhitePapers/OptimizingPa tientFlowMovingPatientsSmoothlyThroughAcuteCareSettings.aspx [Accessed 6 August 2019].

24 Yasunaga $\mathrm{H}$, Miyata $\mathrm{H}$, Horiguchi $\mathrm{H}$, et al. Population density, callresponse interval, and survival of out-of-hospital cardiac arrest. Int $J$ Health Geogr 2011;10:26-9.

25 Committee on the Future of Emergency Care in the United States Health System Board on Health Care Services loM. Emergency medical services at the crossroads. Washington D.C: The National Academies Press, 2006: 309.

26 Sasson C, Rogers MAM, Dahl J, et al. Predictors of survival from outof-hospital cardiac arrest: a systematic review and meta-analysis. Circ Cardiovasc Qual Outcomes 2010;3:63-81.

27 Chocron R, Loeb T, Lamhaut L, et al. Ambulance density and outcomes after out-of-hospital cardiac arrest: insights from the Paris sudden death expertise center registry. Circulation 2019;139:1262-71.

28 Finnegan JR, Meischke H, Zapka JG, et al. Patient delay in seeking care for heart attack symptoms: findings from focus groups conducted in five U.S. regions. Prev Med 2000;31:205-13.

29 Skolarus LE, Murphy JB, Zimmerman MA, et al. Individual and community determinants of calling 911 for stroke among African Americans in an urban community. Circ Cardiovasc Qual Outcomes 2013;6:278-83.

30 Watts J, Cowden JD, Cupertino AP, et al. 911 (nueve once): Spanishspeaking parents' perspectives on prehospital emergency care for children. J Immigr Minor Health 2011;13:526-32.

31 Sasson C, Haukoos JS, Ben-Youssef L, et al. Barriers to calling 911 and learning and performing cardiopulmonary resuscitation for residents of primarily Latino, high-risk neighborhoods in Denver, Colorado. Ann Emerg Med 2015;65:e2:545-52.
32 McCarthy JJ, Carr B, Sasson C, et al. Out-Of-Hospital cardiac arrest resuscitation systems of care: a scientific statement from the American heart association. Circulation 2018;137:e645-60.

33 Hohmann AA, Shear MK. Community-based intervention research: coping with the "noise" of real life in study design. Am J Psychiatry 2002;159:201-7.

34 Bate P, Mendel P, Robert G. Organizing for quality: the improvement journeys of leading hospitals in Europe and the United States. Boca Raton, FL: CRC Press, 2007.

35 Greenhalgh T, Robert G, Macfarlane F, et al. Diffusion of innovations in service organizations: systematic review and recommendations. Milbank Q 2004;82:581-629.

36 Coute RA, Shields TA, Cranford JA, et al. Intrastate variation in treatment and outcomes of out-of-hospital cardiac arrest. Prehosp Emerg Care 2018;22:743-52.

37 CARES. Cares fact sheet. Available: https://mycares.net/sitepages/ factsheet.jsp [Accessed 22 Aug 2019].

38 Michigan CARES. MI-CARES Bi-Annual population assessment. In: CARtE S, ed, 2016.

39 McNally B, Robb R, Mehta M, et al. Out-of-hospital cardiac arrest surveillance --- Cardiac Arrest Registry to Enhance Survival (CARES), United States, October 1, 2005--December 31, 2010. MMWR Surveill Summ 2011;60:1-19.

40 MaxQDA. Discover MaxQDA. Available: https://www.maxqda.com/ what-is-maxqda [Accessed 22 Aug 2019].

41 Miles MB, Huberman AM, Huberman MA, et al. Qualitative data analysis: an expanded Sourcebook. thousand oaks. CA: SAGE, 1994.

42 Nallamothu BK, Guetterman TC, Harrod M, et al. How do resuscitation teams at Top-Performing hospitals for in-hospital cardiac arrest succeed? A qualitative study. Circulation 2018;138:154-63.

43 Bradley EH, Herrin J, Wang Y, et al. Strategies for reducing the door-to-balloon time in acute myocardial infarction. $N$ Engl $J$ Med 2006;355:2308-20.

44 Bradley EH, Nallamothu BK, Herrin J, et al. National efforts to improve door-to-balloon time results from the door-to-balloon alliance. J Am Coll Cardiol 2009;54:2423-9.

45 Nallamothu BK, Bradley EH, Krumholz HM. Time to treatment in primary percutaneous coronary intervention. $N$ Engl $\mathrm{J}$ Med 2007;357:1631-8.

46 Nallamothu BK, Krumholz HM, Peterson ED, et al. Door-To-Balloon times in hospitals within the get-with-the-guidelines registry after initiation of the door-to-balloon (D2B) alliance. Am J Cardiol 2009;103:1051-5.

47 Bradley EH, Curry LA, Spatz ES, et al. Hospital strategies for reducing risk-standardized mortality rates in acute myocardial infarction. Ann Intern Med 2012;156:618-26.

48 Curry LA, Spatz E, Cherlin E, et al. What distinguishes topperforming hospitals in acute myocardial infarction mortality rates? A qualitative study. Ann Intern Med 2011;154:384-90.

49 Chan PS, Nallamothu BK. Improving outcomes following in-hospital cardiac arrest: life after death. JAMA 2012;307:1917-8.

50 Mendel P, Meredith LS, Schoenbaum M, et al. Interventions in organizational and community context: a framework for building evidence on dissemination and implementation in health services research. Adm Policy Ment Health 2008;35:21-37. 